\title{
小麦根系在碱胁迫下的生理代谢反应
}

\author{
郭瑞 $1,2^{*}$ 周 际 ${ }^{3}$ 杨 帆 ${ }^{4}$ 李 峰 ${ }^{1}$ \\ ${ }^{1}$ 中国农业科学院农业环境与可持续发展研究所, 北京 $100081 ;{ }^{2}$ 农业部旱作节水农业重点实验室, 北京 $100081 ;{ }^{3}$ 国土资源部土地整治中心, 北京 \\ $100034 ;{ }^{4}$ 吉林省农业科学院, 长春 130033
}

摘 要 为明确碱胁迫对小麦(Triticum aestivum)根系离子组及代谢组的影响, 探讨其响应变化规律及机制, 该研究以小麦为 实验材料, 采用两种碱性盐 $\left(\mathrm{NaHCO}_{3}\right.$ 和 $\left.\mathrm{Na}_{2} \mathrm{CO}_{3}\right)$ 按摩尔比 $1: 1$ 混合模拟不同碱胁迫强度, 利用气相色谱一质谱联用(GC-MS)技 术结合多元变量分析方法, 系统分析小麦根系在碱胁迫下的矿质元素、游离阴离子、代谢产物及代谢途径变化。结果显示: 低 浓度碱胁迫下小麦根系仍能维持一定的生长, 但在高浓度碱胁迫下根系生长受到了明显抑制。当碱胁迫强度超过小麦根系调 节能力时, 根系中 $\mathrm{Na}$ 含量急剧增加的同时 $\mathrm{K}$ 含量明显减少。碱胁迫刺激根中 $\mathrm{Ca}$ 积累, 而 $\mathrm{Mg} 、 \mathrm{Cu}$ 和 $\mathrm{Fe}$ 含量呈现下降趋势。碱 胁迫明显减少根中游离阴离子(主要是 $\mathrm{Cl}^{-}$) 含量。检测代谢物组包括有机酸、氨基酸、碳水化合物、嘧啶和嘌呤等 70 个代谢产 物, 主成分分析结果表明代谢物均分布在 $95 \%$ 的置信区间内。碱胁明显迫促进苹果酸、琥珀酸等代谢物积累, 但造成糖类(果 糖、蔗糖)及多元醇(肌醇、山梨糖醇)和氨基酸 $\left(\gamma\right.$-氨基丁酸、丙氨酸)含量显著下降。结果表明: 根系中 $\mathrm{Na}^{+}$含量剧增, 加上高 $\mathrm{pH}$ 值危害, 导致根系生长率降低; 与此同时, 游离阴离子明显减少, 造成根系内负电荷亏缺和 $\mathrm{pH}$ 不稳定, 导致离子平衡遭到 破坏, 进而引起一系列代谢途径的协变反应。小麦根系在碱胁迫下糖酵解、细胞膜脂代谢和氨基酸合成受到明显的抑制, 但 三羧酸循环显著增强。这些结果表明碱胁迫(高 $\mathrm{pH}$ 值)对碳素合成和储存有明显的负效应，降低代谢合成碳骨架和能量，使得 清除活性氧能力明显下降。碱胁迫下根外部质子缺乏造成 $\mathrm{NO}_{3}{ }^{-}$含量降低, 影响氮素吸收利用, 导致氨基酸合成受阻。三羧酸 循环增强为生成有机酸类化合物和调控 $\mathrm{pH}$ 平衡提供能量, 这可能是植物适应碱胁迫的一种特殊对应策略。

关键词 小麦; 碱胁迫; 根系; 生长特性; 代谢调控

引用格式: 郭瑞, 周际, 杨帆, 李峰 (2017). 小麦根系在碱胁迫下的生理代谢反应. 植物生态学报, 41, 683-692. doi: 10.17521/cjpe.2016.0136

\section{Metabolic responses of wheat roots to alkaline stress}

GUO Rui ${ }^{12^{*}}$, ZHOU Ji $^{3}$, YANG Fan ${ }^{4}$, and LI Feng ${ }^{1}$

${ }^{1}$ Institute of Environment and Sustainable Development in Agriculture, Chinese Academy of Agricultural Sciences, Beijing 100081, China; ${ }^{2}$ Key Laboratory of Dryland Agriculture, Ministry of Agriculture, Beijing 100081, China; ${ }^{3}$ Land Consolidation and Rehabilitation Centre, the Ministry of Land and Resources, Beijing 100034, China; and ${ }^{4}$ Jilin Academy of Forestry Science, Changchun 130033, China

\section{Abstract}

Aims The aim of this study was to investigate the effects of alkaline stress on primary, secondary metabolites and metabolic pathways in the roots of wheat (Triticum aestivum). The results were used to evaluate the physiological adaptive mechanisms by which wheat tolerated alkali stress.

Methods A pot experiment was carried out in the greenhouse. For each plastic pot, five wheat seeds were planted. After germination, seedlings were allowed to grow under controlled water and nutrient conditions for two months, then seedlings were exposed to alkaline stress $\left(\mathrm{NaHCO}_{3}-\mathrm{Na}_{2} \mathrm{CO}_{3}\right)$ for 12 days. The relative growth rate $(R G R)$, absolute water content $(A W C)$, metal elements, free cations and metabolites were measured.

Important findings The alkaline stress caused the reduction of $R G R$ and AWC. Alkaline stress caused a rapid increase of $\mathrm{Na}$ content with the concurrent decrease in $\mathrm{K}$ and $\mathrm{Cl}$ content, resulting in inhibited metal element accumulation and an ionic imbalance. In the present study, alkaline stress strongly enhanced Ca accumulation in wheat roots, suggesting that an increased $\mathrm{Ca}$ concentration can immediately trigger the salt overly sensitive (SOS)-Na exclusion system and reduce Na-associated injuries. Also, 70 metabolites, including organic acids, amino acids, sugars/polyols and others, behaved differently in the alkaline stress treatments according to a GC-MS analysis. The metabolic profiles of wheat were closely associated with alkaline-stress conditions. Alkaline stress caused the accumulation of organic acids, accompanied by the depletion of sugars/polyols and amino acids. Organic acids could play a central role in the regulation of intracellular $\mathrm{pH}$ by accumulating vacuoles to neutralize

收稿日期Received: 2016-04-14 接受日期Accepted: 2017-04-05

* E-mail: guorui01@caas.cn 
excess cations. Glycolysis and amino acid synthesis in roots were inhibited under salt stress while prolonged alkaline stress led to a progressive tricarboxylic acid (TCA) cycle. The severe negative effects of alkaline stress on sugar synthesis and storage may reflect the toxic levels of $\mathrm{Na}^{+}$accumulating in plant cells in a high-pH environment, implying that the reactive oxygen species detoxification capacity was diminished by the high $\mathrm{pH}$. A lack of $\mathrm{NO}_{3}^{-}$in wheat roots can decrease synthase enzyme activities, limiting the synthesis of amino acids. Under salt stress, the TCA cycle and organic acid accumulation increased, but glycolysis and amino acid synthesis were inhibited in roots. Thus, energy levels and high concentrations of organic acids may be the key adaptive mechanisms by which wheat seedlings maintain their intracellular ion balance under alkaline stress.

Key words wheat (Triticum aestivum); alkaline stress; roots; growth characters; metabolic profiles

Citation: Guo R, Zhou J, Yang F, Li F (2017). Metabolic responses of wheat roots to alkaline stress. Chinese Journal of Plant Ecology, 41, 683-692. doi: 10.17521/cjpe.2016.0136

全球可利用农田面积为 8300 万 $\mathrm{hm}^{2}$ ，其中 800 万 $\mathrm{hm}^{2}$ 灌溉和干旱农田受到了盐碱胁迫的影响。由 于全球人口的快速增长与粮食短缺矛盾日益加深, 土地盐碱化成为影响粮食生产和粮食安全的主要限 制因素(FAO，2005)。我国盐碱化土地面积约为667 万 $\mathrm{hm}^{2}$, 已经成为限制我国农业经济发展的重要因 素(李涁等, 2005)。因此, 提高作物的抗盐碱性, 培 育耐盐碱作物新品种, 将是减少农业作物损失的重 要途径之一, 也是当前农业科学研究的热点。

根据土壤所含盐分特点, 以中性盐 $\mathrm{NaCl}$ 和 $\mathrm{Na}_{2} \mathrm{SO}_{4}$ 为主的称为盐土, 以碱性盐 $\mathrm{NaHCO}_{3}$ 和 $\mathrm{Na}_{2} \mathrm{CO}_{3}$ 为主的称为碱土, 两种土壤往往相伴发生, 想要真正地解决土壤碱化问题, 就必须区别研究盐 胁迫和碱胁迫(俞仁培, 1999; 李涁等, 2005)。碱土主 要是碱性胁迫, $\mathrm{Na}^{+} 、 \mathrm{Cl}^{-} 、 \mathrm{HCO}_{3}^{-}$和 $\mathrm{CO}_{3}{ }^{2-}$ 为主要毒害 离子, 包括渗透胁迫、离子毒害和高 $\mathrm{pH}$ 伤害( $\mathrm{Shi}$ \& Wang, 2005; Yang et al., 2008)。目前, 植物耐碱、 抗碱方面的研究逐渐成为热点, Wang等(2012)认为 碱胁迫对水稻(Oryza sativa)老叶片的影响远大于新 叶, 大量的 $\mathrm{Na}^{+}$和 $\mathrm{Cl}^{-}$堆积在老叶中, 离子平衡被打 破, 同时影响植物矿质元素的吸收, 新、老叶在氮代 谢方面着明显不同。萨如拉等(2014)研究发现燕麦 (Avena sativa) 是通过提高阳离子选择吸收及分配来 适应碱胁迫。Shi等(2015)研究证实碱胁迫对豆科植 物光合作用的危害显著大于盐胁迫, 碱胁迫造成叶 片光合色素含量降低, 抑制气体交换能力进而危害 植物体光合同化功能。目前研究主要集中在植物对 碱胁迫生理、生态响应, 少数涉及植物耐抗碱分子 机制, 对植物通过自身代谢网络调控来缓解伤害适 应胁迫的系统研究更少。

本试验通过研究不同碱胁迫条件下小麦 (Triticum aestivum)根系矿质元素吸收, 离子平衡以
及次生代谢产物的变化特点, 揭示小麦根系对高 $\mathrm{pH}$ 的特殊调节作用，同时绘制代谢网络通路图，阐明 碱胁迫下小麦根系代谢网络调控的内在机制, 为从 分子水平研究小麦抗碱机制提供理论依据。

\section{1 材料和方法}

\section{1 实验材料和模拟胁迫}

小麦品种“石家庄 8 ”号，千粒重约为 $43 \mathrm{~g}$, 全生 育期约 237 天，具有较好的抗盐碱性。

根据我国北方盐碱化土壤主要盐分组成，盐 度、碱度复杂多变的特点, 以及小麦耐碱性特点, 分 别选取 $\mathrm{NaHCO}_{3}$ 和 $\mathrm{Na}_{2} \mathrm{CO}_{3}$ 2种碱性盐并按摩尔比 $1: 1$ 混合，模拟 50 和 $100 \mathrm{mmol} \cdot \mathrm{L}^{-1} 2$ 个碱胁迫强度。利用 便携式DDG-2080-S 电导仪 (博取公司, 上海)、 Wescor Psypro露点水势仪(Wescor, Utah，USA)和 PHS-3C型数字 $\mathrm{pH}$ 计(虹益公司, 上海)测定处理液的 电导率、水势和 $\mathrm{pH}$ 值。

\section{2 材料培养}

挑选整齐一致的小麦种子播种于直径 $17 \mathrm{~cm}$ 的塑料花盆内, 每盆装 $2.5 \mathrm{~kg}$ 洗净的细砂。共种植 20盆，每盆定苗5株，出苗后每天17:00-18:00用 $\mathrm{pH}$ 值为 6.95 的 0.5 倍Hoagland营养液 $\left(5.00 \mathrm{mmol} \cdot \mathrm{L}^{-1}\right.$ $\mathrm{Ca}^{2+}, 2.00 \mathrm{mmol} \cdot \mathrm{L}^{-1} \mathrm{Mg}^{2+}, 6.04 \mathrm{mmol} \cdot \mathrm{L}^{-1} \mathrm{~K}^{+}, 2.22 \times$ $10^{-2} \mathrm{mmol} \cdot \mathrm{L}^{-1}$ EDTA-Fe ${ }^{2+}, 6.72 \times 10^{-3} \mathrm{mmol} \cdot \mathrm{L}^{-1}$ $\mathrm{Mn}^{2+}, 3.16 \times 10^{-4} \mathrm{mmol} \cdot \mathrm{L}^{-1} \mathrm{Cu}^{2+}, 7.65 \times 10^{-4} \mathrm{mmol} \cdot \mathrm{L}^{-1}$ $\mathrm{Zn}^{2+}, 2.10 \mathrm{mmol} \cdot \mathrm{L}^{-1} \mathrm{SO}_{4}{ }^{2-}, 1.00 \mathrm{mmol} \cdot \mathrm{L}^{-1} \mathrm{H}_{2} \mathrm{PO}_{4}^{-}$, $4.63 \times 10^{-2} \mathrm{mmol} \cdot \mathrm{L}^{-1} \mathrm{H}_{3} \mathrm{BO}_{3}, 5.56 \times 10^{-4} \mathrm{mmol} \cdot \mathrm{L}^{-1}$ $\left.\mathrm{H}_{2} \mathrm{MoO}_{4}, 15.04 \mathrm{mmol} \cdot \mathrm{L}^{-1} \mathrm{NO}_{3}^{-}\right)$进行一次透灌。生长 2 个月后, 幼苗株高约 $(34.22 \pm 3.72) \mathrm{cm}$, 将 20 盆分 为 4 组, 每组为 5 盆, 1 盆为 1 个重复。其中有 1 组作为 胁迫处理前, 基础生物量指标的测试样品。1组为对 照组, 每天仍浇 0.5 倍Hoagland营养液; 其他 2 组按

www.plant-ecology.com 
照模拟碱胁迫对小麦进行连续 12 天的处理。整个实 验在温室内进行, 白天温度设定在 $(25 \pm 1){ }^{\circ} \mathrm{C}$, 夜 间温度设定在 $(19 \pm 1){ }^{\circ} \mathrm{C}$, 温室内光照均匀 $(700$ $\left.\mu \mathrm{mol} \cdot \mathrm{m}^{-2} \cdot \mathrm{s}^{-1}\right)$, 每日光照时间为 $15 \mathrm{~h}$ 。

\section{3 取样}

碱胁迫处理结束后, 将植株保持完整根系取 出, 将根与地上部分分开, 用蒸馏水洗净根系, 并 吸干多余水分, 称取根系鲜样用于测定生理指标。 将 $100 \mathrm{mg}$ 根系鲜样液氮速冻, $-80{ }^{\circ} \mathrm{C}$ 冰箱冷藏用于 代谢物的测定。剩余材料置 $105{ }^{\circ} \mathrm{C}$ 烘箱内杀青 $5 \mathrm{~min}$, 随后真空低温干燥至恒质量, 称取干质量。将样品 磨成粉末，用于测定金属元素和游离阴离子含量。

\section{4 生理指标测定}

\subsection{1 生长指标的测定}

根系相对生长率 $(R G R)=(\ln$ 处理后干质量 $-\ln$ 处理前干质量)/处理天数(Kingsbury et al., 1984)

根系绝对含水量 $(A W C)=($ 鲜质量-干质量) /鲜 质量, 单位为 $\mathrm{mg} \cdot \mathrm{g}^{-1}$ 。

\subsection{2 矿质元素和游离阴离子指标的测定}

利用DX-300离子色谱仪(Thermo Fisher Scientific, Haverhill, USA)、AS4A-SC 色谱柱 (Dionex, Sunnyvale, USA) 和 CDM-II 电导检测器 (Dionex, Sunnyvale, USA), $\mathrm{Na}_{2} \mathrm{CO}_{3} / \mathrm{NaHCO}_{3}=1.7 / 1.8 \mathrm{mmol} \cdot \mathrm{L}^{-1}$ 的混合溶液作为流动相, 测定根系中矿质元素 $\mathrm{K}$ 、

$\mathrm{Na} 、 \mathrm{Ca} 、 \mathrm{Mg} 、 \mathrm{Cu} 、 \mathrm{Fe} 、 \mathrm{Zn} 、 \mathrm{Mn}$ 及游离阴离子 $\mathrm{NO}_{3}^{-}$、 $\mathrm{Cl}^{-} 、 \mathrm{SO}_{4}{ }^{2-} 、 \mathrm{H}_{2} \mathrm{PO}_{4}^{-}$。

\subsection{3 代谢物检测和篮选的测定}

萃取衍生化: 取 $100 \mathrm{mg}$ 小麦根系样本放入 $2 \mathrm{~mL}$ 离心管里, 加入 $60 \mu \mathrm{L}$ 核糖醇漩浴混匀; 再加入 $0.35 \mathrm{~mL}$ 甲醇漩浴混匀。 $70 \mathrm{~Hz}$ 研磨 $5 \mathrm{~min}$, 在 $70{ }^{\circ} \mathrm{C}$ 烘箱中放置 $10 \mathrm{~min}$; 紧接着将样本离心 $10 \min \left(4^{\circ} \mathrm{C}\right.$, $12000 \mathrm{r} \cdot \mathrm{min}^{-1}$ ); 取出 $0.35 \mathrm{~mL}$ 上清液放入 $2 \mathrm{~mL}$ 进 样瓶中(甲烷硅基化)。在真空浓缩器中干燥提取物 $\left(45{ }^{\circ} \mathrm{C}, 1.5 \mathrm{~h}\right)$; 向干燥后的代谢物中加入 $80 \mu \mathrm{L}$ 甲氧 胺盐试剂(甲氧胺盐酸盐, 溶于吡啶 $20 \mathrm{mg} \cdot \mathrm{mL}^{-1}$ ), 轻轻混匀后, 放入烘箱中 $37{ }^{\circ} \mathrm{C}$ 捊育 $2 \mathrm{~h}$; 向每个样 品中迅速加入 $100 \mu \mathrm{L} \mathrm{N}, \mathrm{O}$-双(三甲基硅烷基)三氟乙 酰胺BSTFA (含有 $1 \%$ 三甲基氯硅烷(TCMS), v/v), 将 混合物 $70{ }^{\circ} \mathrm{C}$ 下反应 $1 \mathrm{~h}$, 冷却至室温(Lisec et al., 2006)。

上机检测: 使用Agilent 7890气相色谱仪质谱 联机(Agilent, Palo Alto, USA)进行 GC-MS分析。
GC-MS 分析条件：DN-5MS毛细管柱 $(30 \mathrm{~m} \times$ $250 \mu \mathrm{m} \times 0.25 \mu \mathrm{m}, \mathrm{J} \& \mathrm{~W}$ Scientific, Folsom, USA); 进样量 $1 \mu \mathrm{L}$; 载气: 氦气; 前进样口吹扫流速: $3 \mathrm{~mL} \cdot \mathrm{min}^{-1}$; 流速: $1 \mathrm{~mL} \cdot \mathrm{min}^{-1}$; 柱温: 初始温度 $90{ }^{\circ} \mathrm{C}$ 保持 $15 \mathrm{~s}$, 以 $10{ }^{\circ} \mathrm{C} \cdot \mathrm{min}^{-1}$ 升至 $180{ }^{\circ} \mathrm{C}$, 再以 $5{ }^{\circ} \mathrm{C} \cdot \mathrm{min}^{-1}$ 升至 $240{ }^{\circ} \mathrm{C}$, 再以 $20{ }^{\circ} \mathrm{C} \cdot \mathrm{min}^{-1}$ 继续升至 $285{ }^{\circ} \mathrm{C}$ 保持 $11.5 \mathrm{~min}$ 。前进样口温度, 传输线温度和 离子源温度分别为 $280 、 245$ 和 $220{ }^{\circ} \mathrm{C}$ 。电离电压: $-70 \mathrm{eV}$; 扫描方式(全扫描): $20-600 \mathrm{~m} \cdot \mathrm{z}^{-1}$; 扫描速 度: 100 spectra $\cdot \mathrm{s}^{-1}$; 溶剂延迟: $492 \mathrm{~s}$ 。

定性定量: 将所得的 GC-MS 原始数据采取 LECO 公司的 Chroma TOF4.3X软件和 LECO-Fiehn Rtx5数据库, 进行峰定性和定量, 使用内标标准化法 处理导出的数据。利用SIMCA 14.0软件进行分析。

\section{5 数据分析}

利用SPSS 17.0和Microsoft Excel对生长和阴阳 离子指标进行数据统计和分析, 试验数据以平均值 \pm 标准误差表示, 每个数据均是 5 次重复的平均值, $p<0.05$ 被视为显著性差异。利用SIMCA 14.0对代谢 产物进行分析, 在主成分分析(PCA)中, 使用均值 中心化 (mean-centered) 对数据进行标准化处理, 结 果分别用得分(scores)和载荷(loading)表示。正交偏 最小二乘判别式(OPLS-DA)自动规格化标准化处理 进一步分析两组间区分贡献显著的代谢物。采用 $\log _{2}$ (胁迫/对照)倍数变化分析胁迫对代谢物的影响, 正值代表含量升高, 负值代表含量降低。根据相似 度 $>700$ 、VIP (variable importance in the projection) $>$ 1、 $p<0.05$ 和 $p<0.01$ 对代谢差异物进行鉴定。

\section{2 结果}

\section{1 处理液电导率、 $\mathrm{pH}$ 和水势值}

表1表明, 随碱浓度增加, 处理液电导率大幅 度上升, 渗透势大幅度下降。碱胁迫处理液的 $\mathrm{pH}$ 值 明显大于对照液。

表1 不同盐浓度处理液电导率、 $\mathrm{pH}$ 值和水势值

Table 1 The electrical conductivity $(E C), \mathrm{pH}$ value and osmotic potential of salinity stress treatment solutions

\begin{tabular}{lllll}
\hline $\begin{array}{l}\text { 处理 } \\
\text { Treatment }\end{array}$ & $\begin{array}{l}\text { 碱浓度 } \\
\text { Alkaline } \\
\text { concentration } \\
\left(\mathrm{mmol} \cdot \mathrm{L}^{-1}\right)\end{array}$ & $\begin{array}{l}\text { 电导率 } \\
\mathrm{EC}\left(\mathrm{dS} \cdot \mathrm{m}^{-1}\right)\end{array}$ & $\begin{array}{l}\text { 渗透势 } \\
\text { Osmotic } \\
\text { potential } \\
(\mathrm{MPa})\end{array}$ & $\begin{array}{l}\mathrm{pH} \text { 值 } \\
\mathrm{pH} \text { value }\end{array}$ \\
\hline 对照 Control & 0 & 2.16 & -0.05 & 6.95 \\
$\begin{array}{l}\text { 碱胁迫 } \\
\text { Alkaline stress }\end{array}$ & 50 & 4.98 & -0.28 & 9.69 \\
& 100 & 9.21 & -0.51 & 9.92 \\
\hline
\end{tabular}

doi: $10.17521 /$ cjpe. 2016.0136 


\section{2 碱胁迫对小麦生长特性的影响}

碱胁迫下根系 $R G R$ 随盐浓度增加而下降, 且下 降幅度随盐浓度增加而加强(图1A, $p<0.05$ )。根系 $A W C$ 变化趋势和 $R G R$ 基本相同, 胁迫强度越高下降 越显著(图1B, $p<0.05$ )。

\section{3 碱胁迫对小麦矿质元素吸收和游离阴离子的 影响}

如表2所示，在碱胁迫 12 天后小麦根系中矿质 元素含量发生了显著变化。与对照相比较, 碱胁迫 下小麦根系中 $\mathrm{Na}$ 元素含量随盐度增加呈现上升
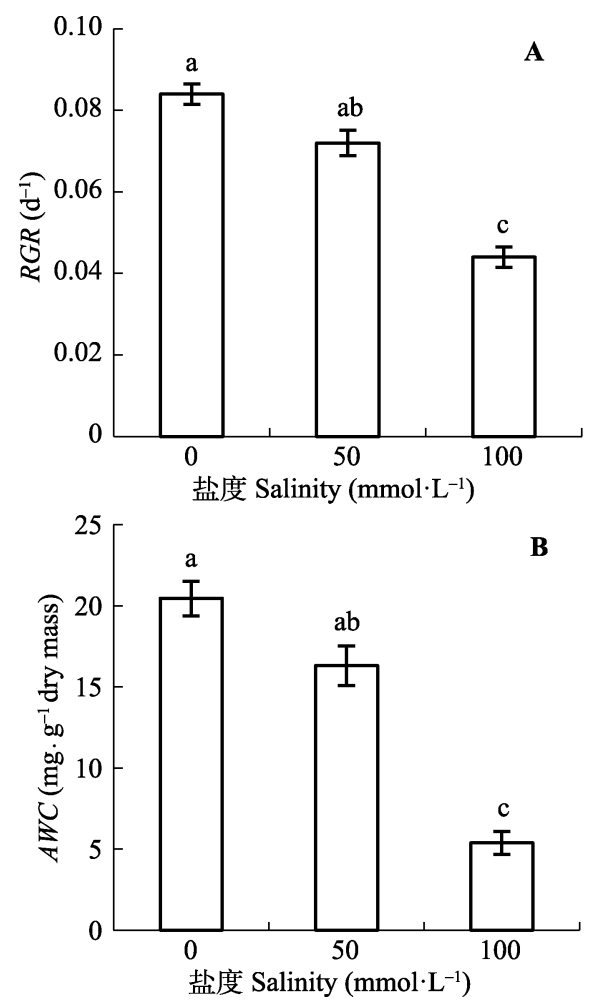

图1 碱浓度对小麦根系相对生长率 $(R G R)$ 和绝对含水量 $(A W C$ )的影响(平均值 \pm 标准误差, $n=5$ )。不同小写字母表示 不同处理间差异显著 $(p<0.05)$ 。

Fig. 1 Effects of alkaline concentration on root relative growth rate $(R G R)$ and absolute water content (AWC) (mean \pm $S E, n=5$ ). Different lowercase letters indicate significant differences among the various treatments $(p<0.05)$.
趋势，但 $\mathrm{K}$ 元素含量呈现下降趋势，且随着盐度的 增加变化幅度加强(表 $2, p<0.05$ )。当盐度为 50 $\mathrm{mmol} \cdot \mathrm{L}^{-1}$ 时根系中 $\mathrm{Ca}$ 含量缓慢增加, 但盐度达到 $100 \mathrm{mmol} \cdot \mathrm{L}^{-1}$ 后 $\mathrm{Ca}$ 含量急剧增加(表 $2, p<0.05$ )。 根系中 $\mathrm{Mg} 、 \mathrm{Cu} 、 \mathrm{Fe} 、 \mathrm{Zn}$ 元素对碱胁迫的响应相似，都 是随着盐度的增加, 含量呈现下降趋势, 但在中低 碱胁迫下 $\mathrm{Cu}$ 和 $\mathrm{Fe}$ 变化不明显(表 $2, p<0.05$ )。另外, 碱胁迫对 $\mathrm{Mn}$ 元素的影响不明显(表 $2, p<0.05$ )。碱胁 迫下小麦根系 $\mathrm{Cl}^{-}$和 $\mathrm{NO}_{3}{ }^{-}$含量均下降, 且随着盐度 增加下降幅度增大(表 $3, p<0.05)$ 。碱胁迫下根系中 $\mathrm{H}_{2} \mathrm{PO}_{4}{ }^{-}$含量均低于对照处理组(表3, $p<0.05$ )。而碱 胁迫对根系中 $\mathrm{SO}_{4}{ }^{2-}$ 含量影响不大 $($ 表 $3, p<0.05$ )。

\section{4 碱胁迫下小麦根系代谢组变化轨迹}

本研究利用 GC-MS技术检测小麦根系对碱 胁迫的生理响应过程, 主成分分析 (PCA)的得分 图显示, 3组样本一一对照组 $\left(0 \mathrm{mmol} \cdot \mathrm{L}^{-1}\right)$ 、中度 碱胁迫 $\left(50 \mathrm{mmol} \cdot \mathrm{L}^{-1}\right)$ 和重度碱胁迫 $\left(100 \mathrm{mmol} \cdot \mathrm{L}^{-1}\right)$ 呈现显著性差异，两个主成分得分为 $95 \%$ ，这表明 本研究 GC-MS的代谢组学方法可以有效地表征小 麦根系碱胁迫下的生理代谢状态(图2A)。OPLS-DA 结果进一步证实小麦根系对照组和碱胁迫组代谢发 生显著性变化, 得分图显示对照组分别和 $50 \mathrm{mmol} \cdot \mathrm{L}^{-1}$ 碱胁迫处理(建模有效性得分值 $\left(R^{2} X\right)=0.86, Q^{2}=$ $0.99)$ 以及 $100 \mathrm{mmol} \cdot \mathrm{L}^{-1}$ 碱胁迫处理 $\left(R^{2} X=0.91, Q^{2}=\right.$ 0.99)都得到了很好的OPLS-DA模型(图2B、2C)。

\section{5 碱胁迫对小麦根系代谢产物的影响}

根据主成分分析结果, 在 $50 \mathrm{mmol} \cdot \mathrm{L}^{-1}$ 和 100 $\mathrm{mmol} \cdot \mathrm{L}^{-1}$ 碱胁迫下小麦根系与对照相比较, 分别 有 33 个和 57 个代谢物发生显著性变化, 其中相同的 代谢物在高碱胁迫下变化倍数明显高于中低碱胁迫 (表 $4, p<0.05 ; p<0.01$ )。如表4显示, 在三羧酸(TCA) 循环中不同浓度碱胁迫均促进柠檬酸、琥珀酸和苹 果酸的合成, 另外在 $100 \mathrm{mmol} \cdot \mathrm{L}^{-1}$ 碱胁迫下其他参 与 $\mathrm{TCA}$ 循环的代谢物都呈现极显著的上升(表4,

表2 碱胁迫对小麦根系干物质中矿质元素含量的影响(平均值土标准误差, $n=5$ )

Table 2 Effects of alkaline stress on the contents of metal elements in the roots of wheat seedlings $(\operatorname{mean} \pm S E, n=5)$

\begin{tabular}{|c|c|c|c|c|c|c|c|c|c|}
\hline \multirow{2}{*}{$\begin{array}{l}\text { 处理 } \\
\text { Treatment }\end{array}$} & \multirow{2}{*}{$\begin{array}{l}\text { 碱胁迫浓度 } \\
\text { Alkaline concentration } \\
(\mathrm{mmol})\end{array}$} & \multicolumn{8}{|c|}{ 矿质元素 Metal elements (mmol· $\cdot \mathrm{g}^{-1}$ dry mass) } \\
\hline & & $\mathrm{Na}$ & $\mathrm{K}$ & $\mathrm{Ca}$ & $\mathrm{Mg}$ & $\mathrm{Cu}$ & $\mathrm{Fe}$ & $\mathrm{Zn}$ & $\mathrm{Mn}$ \\
\hline 对照 Control & 0 & $4.25 \pm 1.01^{\mathrm{b}}$ & $69.90 \pm 3.03^{\mathrm{a}}$ & $17.11 \pm 1.01^{b}$ & $7.80 \pm 0.29^{\mathrm{a}}$ & $1.25 \pm 0.04^{\mathrm{a}}$ & $1.56 \pm 0.08^{\mathrm{a}}$ & $0.07 \pm 0.01^{\mathrm{a}}$ & $0.06 \pm 0.00^{\mathrm{a}}$ \\
\hline \multirow{2}{*}{$\begin{array}{l}\text { 碱胁迫 } \\
\text { Alkaline stress }\end{array}$} & 50 & $15.89 \pm 2.06^{\mathrm{ab}}$ & $45.20 \pm 1.54^{b}$ & $20.53 \pm 1.00^{\mathrm{b}}$ & $6.01 \pm 0.16^{\mathrm{b}}$ & $1.02 \pm 0.02^{\mathrm{a}}$ & $1.26 \pm 0.03^{\mathrm{a}}$ & $0.05 \pm 0.00^{\mathrm{b}}$ & $0.05 \pm 0.00^{\mathrm{a}}$ \\
\hline & 100 & $19.09 \pm 2.11^{\mathrm{a}}$ & $16.83 \pm 1.02^{\mathrm{c}}$ & $32.85 \pm 1.30^{\mathrm{a}}$ & $4.96 \pm 0.47^{\mathrm{c}}$ & $0.55 \pm 0.03^{\mathrm{b}}$ & $0.84 \pm 0.05^{\mathrm{b}}$ & $0.03 \pm 0.00^{\mathrm{c}}$ & $0.06 \pm 0.00^{\mathrm{a}}$ \\
\hline
\end{tabular}

不同小写字母表示不同处理间差异显著 $(p<0.05)$ 。

Different lowercase letters indicate significant differences among the various treatments $(p<0.05)$.

www.plant-ecology.com 
表3 碱胁迫对小麦根系干物质中阴离子含量的影响(平均值土标准误差, $n=5$ )

Table 3 Effects of alkaline stress on the contents of $\mathrm{Cl}^{-}, \mathrm{NO}_{3}^{-}, \mathrm{H}_{2} \mathrm{PO}_{4}^{-}, \mathrm{SO}_{4}{ }^{2-}$ in the roots of wheat seedlings $($ mean $\pm S E, n=5)$.

\begin{tabular}{|c|c|c|c|c|c|}
\hline \multirow{2}{*}{$\begin{array}{l}\text { 处理 } \\
\text { Treatment }\end{array}$} & \multirow{2}{*}{$\begin{array}{l}\text { 碱胁迫浓度 } \\
\text { Alkaline concentration (mmol) }\end{array}$} & \multicolumn{4}{|c|}{ 阴离子 Anions $\left(\mathrm{mmol} \cdot \mathrm{g}^{-1}\right.$ dry mass) } \\
\hline & & $\mathrm{Cl}^{-}$ & $\mathrm{NO}_{3}^{-}$ & $\mathrm{H}_{2} \mathrm{PO}_{4}^{-}$ & $\mathrm{SO}_{4}{ }^{2-}$ \\
\hline 对照 Control & 0 & $0.12 \pm 0.01^{\mathrm{a}}$ & $0.41 \pm 0.02^{\mathrm{a}}$ & $0.05 \pm 0.00^{\mathrm{a}}$ & $0.03 \pm 0.00^{\mathrm{a}}$ \\
\hline \multirow[t]{2}{*}{ 碱胁迫 Alkaline stress } & 50 & $0.09 \pm 0.00^{\mathrm{b}}$ & $0.34 \pm 0.01^{\mathrm{b}}$ & $0.03 \pm 0.01^{\mathrm{b}}$ & $0.02 \pm 0.00^{\mathrm{a}}$ \\
\hline & 100 & $0.04 \pm 0.01^{\mathrm{c}}$ & $0.07 \pm 0.00^{\mathrm{c}}$ & $0.02 \pm 0.00^{\mathrm{b}}$ & $0.03 \pm 0.00^{\mathrm{a}}$ \\
\hline
\end{tabular}

不同小写字母表示不同处理间差异显著 $(p<0.05)$ 。

Different lowercase letters indicate significant differences among the various treatments $(p<0.05)$.
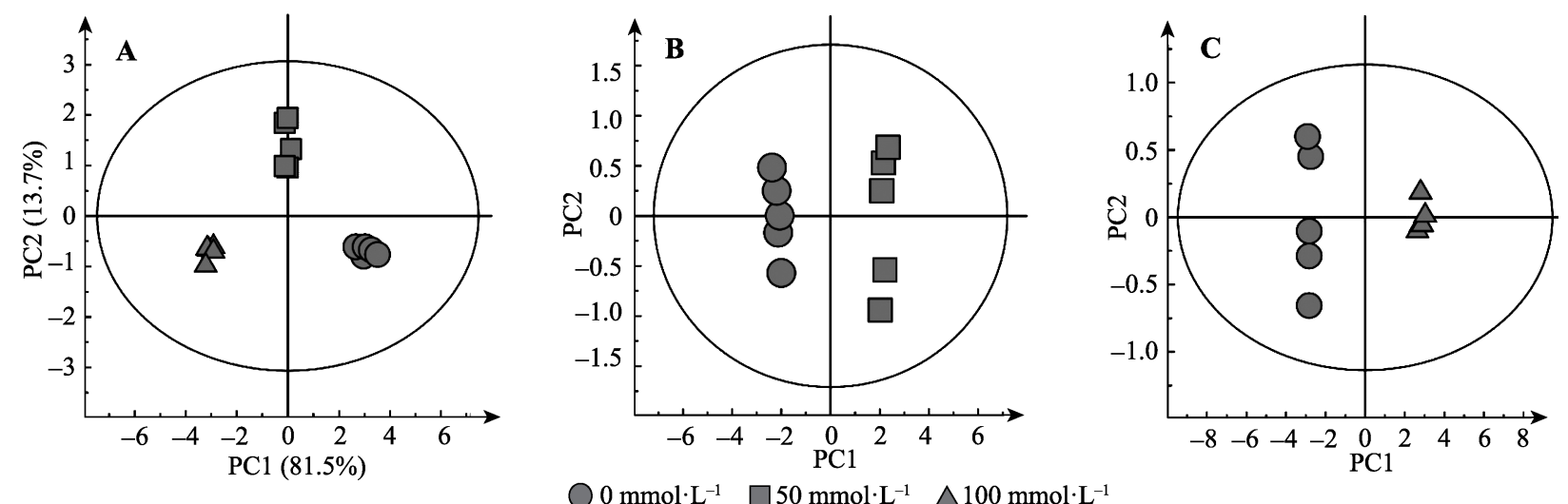

图2 小麦根系在不同碱胁迫强度下代谢变化过程的SIMCA得分图。PCA得分图 (A)。OPLS-DA得分图, 对照组vs 50 $\mathrm{mmol} \cdot \mathrm{L}^{-1}$ 碱胁迫 $(\mathbf{B})$, 对照组 $\mathrm{vs} 100 \mathrm{mmol} \cdot \mathrm{L}^{-1}$ 碱胁迫 $(\mathbf{C})$ 。

Fig. 2 SIMCA analyzed score plots showing the metabolomic trajectory of roots of wheat seedlings under different salinity concentration treatments. Principal component analysis (PCA) score plots (A). Orthogonal partial least squares discriminant analysis (OPLS-DA) scores: CK vs. AS- $50 \mathrm{mmol} \cdot \mathrm{L}^{-1}$ (B) and CK vs. AS- $100 \mathrm{mmol} \cdot \mathrm{L}^{-1}$ (C).

$p<0.05 ; p<0.01)$ 。但是, 碱胁迫迫使参与糖酵解的 代谢物含量降低, 尤其在高盐浓度下(表 $4, p<0.05$; $p<0.01)$ 。不同浓度碱胁迫均造成参与莽草酸途径 的苯丙氨酸和酪氨酸含量降低, $100 \mathrm{mmol} \cdot \mathrm{L}^{-1}$ 浓度 又使得色氨酸和肉桂酸含量降低, 但与此同时莽草 酸和奎尼酸在高碱胁迫下显著增加(表 $4, p<0.05 ; p$ $<0.01)$ 。与对照相比, 碱胁迫使肌醇、甘氨酸、丝 氨酸和乙醇胺含量降低, 这表明碱胁迫明显抑制细 胞膜脂代谢过程，尤其在高 $\mathrm{pH}$ 值条件下(表 $4, p<$ $0.05 ; p<0.01$ )。碱胁迫导致氨基酸显著性减少, 另 外没有引起脯氨酸的显著增加(表 $4, p<0.05 ; p<$ $0.01)$ 。碱胁迫造成小麦根系中糖类和多元醇代谢物 含量降低, 包括葡萄糖、果糖、蔗糖等; 但是明显 引起有机酸类物质含量显著升高, 包括柠檬酸琥珀 酸乳酸等 $(p<0.05 ; p<0.01)$ 。

\section{3 讨论}

\section{1 碱胁迫对小麦生长和矿质元素吸收的影响}

研究已经证明盐胁迫与碱胁迫是不同性质的 胁迫, 且碱胁迫对植物的影响远大于盐胁迫(Shi \&
Wang, 2005; Yang et al., 2008)。一直以来，国内外抗 逆生理多集中在植物抗盐方面的研究, 而对碱胁迫 这一严重的环境问题仍未得到研究者的充分重视 (王晓冬等, 2011; 杨婷等, 2014)。植物相对生长率 $(R G R)$ 和相对含水量 $(W C)$ 是生命活动的最终体现, 它们的变化可以反映植物体受到外界环境胁迫时的 基本生理反应，因此可以作为植物对外界环境胁迫 的响应指标(Yang et al., 2008)。本研究表明当碱胁迫 浓度大于 $50 \mathrm{mmol} \cdot \mathrm{L}^{-1}$ 小麦根系 $R G R$ 受到明显的抑 制, 而贾娜尔 ・阿汗等(2007)研究发现在 $80 \mathrm{mmol} \cdot \mathrm{L}^{-1}$ 碱胁迫下抗盐碱植物碱地肤(Kochia sieversiana)根 系依然保持很高的 $R G R$, 这说明植物的根系对 $\mathrm{pH}$ 的 调节和适应能力, 是决定植物体自身抗碱性的关键 生理特性。碱胁迫导致根系AWC降低, 这可能是由 于碱胁迫导致液泡内质子减少, 破坏细胞膜, 影响 跨膜电化学势梯度, 造成植物大量失水(Yang et al., 2008)。

高等植物细胞质内 $\mathrm{Na}$ 含量较低而 $\mathrm{K}$ 含量较高, 这种状态与渗透调节密切相关(Munns \& Tester, 2008)。由于 $\mathrm{Na}^{+}$和 $\mathrm{K}^{+}$水合半径相似, 因此它们之间 
表4＼cjkstart不同碱胁迫下小麦根系提取物中主要代谢物相对含量和变化倍数

Table 4 Relative concentration and changes of major metabolites in roots of wheat seedlings after alkaline stress treatment

\begin{tabular}{|c|c|c|c|c|c|c|}
\hline \multirow{2}{*}{\multicolumn{2}{|c|}{$\begin{array}{l}\text { 代谢路径和代谢物名称 } \\
\text { Metabolic pathways and metabolites }\end{array}$}} & \multicolumn{3}{|c|}{ 相对含量 Relative concentration } & \multicolumn{2}{|c|}{ 倍性变量Fold changes } \\
\hline & & \multirow{2}{*}{$\begin{array}{c}\mathrm{CK} \\
75.73\end{array}$} & \multirow{2}{*}{$\begin{array}{c}\text { AS-50 mmol } \\
150.14\end{array}$} & \multirow{2}{*}{$\frac{\mathrm{AS}-100 \mathrm{mmol}}{231.23}$} & \multirow{2}{*}{$\frac{\log _{2}(50 / \mathrm{CK})}{0.99^{*}}$} & \multirow{2}{*}{$\frac{\log _{2}(100 / \mathrm{CK})}{1.61^{* *}}$} \\
\hline 三羧酸循环 & 柠檬酸 Citric acid & & & & & \\
\hline $\begin{array}{l}\text { Tricarboxylic acid } \\
\text { cycle }\end{array}$ & 乌头酸 Aconitic acid & 1.34 & 1.70 & 5.13 & 0.34 & $1.93^{* *}$ \\
\hline & $\alpha$-酮戊二酸 $\alpha$-ketoglutaric acid & 0.18 & 0.33 & 0.85 & 0.89 & $2.23^{* *}$ \\
\hline & 琥珀酸 Succinic acid & 19.05 & 61.45 & 120.69 & $1.69^{* *}$ & $2.66^{* *}$ \\
\hline & 延胡索酸 Fumaric acid & 1.34 & 1.56 & 9.63 & 0.23 & $2.85^{* *}$ \\
\hline & 苹果酸 Malic acid & 10.47 & 23.10 & 30.35 & $1.14^{*}$ & $1.54^{* *}$ \\
\hline \multirow{6}{*}{$\begin{array}{l}\text { 糖酵解过程 } \\
\text { Glycolysis }\end{array}$} & 葡萄糖 Glucose & 26.37 & 13.67 & 7.91 & $-0.95^{*}$ & $-1.74^{* *}$ \\
\hline & 葡萄糖-6-磷酸 Fructose-6-phosphate & 0.57 & 0.25 & 0.08 & $-1.16^{*}$ & $-2.75^{* *}$ \\
\hline & 果糖-6-磷酸 Glucose-6-phosphate & 0.22 & 0.13 & 0.04 & -0.80 & $-2.58^{* *}$ \\
\hline & 3-磷酸甘油酸 3-phosphoglyceric acid & 0.50 & 0.34 & 0.11 & -0.57 & $-2.19^{* *}$ \\
\hline & 丙酮酸 Pyruvate & 0.54 & 0.50 & 0.31 & -0.10 & -0.81 \\
\hline & 磷酸烯醇式丙酮酸 Enolphosphopyruvate & 0.73 & 0.61 & 0.22 & -0.26 & $-1.74^{* *}$ \\
\hline \multirow{6}{*}{$\begin{array}{l}\text { 莽草酸途径 } \\
\text { Shikimic path way }\end{array}$} & 莽草酸 Shikimic acid & 1.84 & 1.22 & 3.84 & -0.60 & $1.06^{*}$ \\
\hline & 奎尼酸 Quinic acid & 4.17 & 6.17 & 23.11 & 0.58 & $2.47^{* *}$ \\
\hline & 苯丙氨酸 Phenylalanine & 0.65 & 0.17 & 0.11 & $-1.91^{*}$ & $-2.60^{* *}$ \\
\hline & 色氨酸 Tryptophan & 0.05 & 0.03 & 0.02 & -0.71 & $-1.45^{*}$ \\
\hline & 酪氨酸 Tyrosine & 1.59 & 0.03 & 0.02 & $-4.18^{* *}$ & $-6.57^{* *}$ \\
\hline & 肉桂酸 Cinnamic acid & 0.25 & 0.15 & 0.12 & -0.77 & $-1.12^{*}$ \\
\hline \multirow{4}{*}{$\begin{array}{l}\text { 细胞膜脂代谢 } \\
\text { Metabolism of } \\
\text { plasma membrane }\end{array}$} & 肌醇 Myo-inositol & 19.17 & 9.63 & 3.07 & $-0.99^{*}$ & $-2.64^{* *}$ \\
\hline & 甘氨酸 Glycine & 0.74 & 0.34 & 0.12 & $-1.56^{* *}$ & $-2.69^{* *}$ \\
\hline & 丝氨酸 Serine & 17.83 & 9.56 & 1.64 & $-0.90^{*}$ & $-3.44^{* *}$ \\
\hline & 乙醇胺 Ethanolamine & 20.23 & 11.31 & 3.26 & $-0.84^{*}$ & $-2.63^{* *}$ \\
\hline \multirow{7}{*}{$\begin{array}{l}\text { 氨基酸 } \\
\text { Amino acid }\end{array}$} & $\gamma$-氨基丁酸 $\gamma$-aminobutyric acid & 137.19 & 41.77 & 23.92 & $-1.72^{* *}$ & $-2.52^{* *}$ \\
\hline & 丙氨酸 Alanine & 106.58 & 56.88 & 10.64 & $-0.91^{*}$ & $-3.32^{* *}$ \\
\hline & 谷氨酸 Glutamate & 24.93 & 13.73 & 8.40 & $-0.86^{*}$ & $-1.57^{* *}$ \\
\hline & 天冬酰胺 Asparagine & 12.65 & 4.38 & 0.91 & $-1.53^{* *}$ & $-3.79^{* *}$ \\
\hline & 天冬氨酸 Aspartic acid & 7.60 & 4.06 & 3.34 & $-0.90^{*}$ & $-1.19^{*}$ \\
\hline & 脯氨酸 Proline & 10.04 & 15.47 & 11.57 & 0.62 & 0.20 \\
\hline & 赖氨酸 Lysine & 17.32 & 0.33 & 0.11 & $-5.72^{* *}$ & $-7.26^{* *}$ \\
\hline \multirow{5}{*}{$\begin{array}{l}\text { 糖类及多元醇 } \\
\text { Sugars and polyols }\end{array}$} & 果糖 Fructose & 814.31 & 574.62 & 173.68 & $-0.78^{*}$ & $-2.23^{* *}$ \\
\hline & 蔗糖 Sucrose & 136.11 & 92.04 & 65.17 & -0.56 & $-1.06^{*}$ \\
\hline & 塔罗糖 Talose & 114.17 & 75.07 & 23.13 & -0.60 & $-2.30^{* *}$ \\
\hline & 蔗果三糖 Kestose & 57.63 & 42.47 & 19.69 & -0.44 & $-1.55^{* *}$ \\
\hline & 核糖 Ribose & 8.24 & 4.25 & 1.38 & $-0.96^{*}$ & $-2.58^{* *}$ \\
\hline
\end{tabular}

每个代谢产物的相对含量都是 5 次生物学重复测量的平均值。变化倍数来自于公式 $\log _{2}$ (胁迫/对照), *表示显著性差别 $(p<0.05) ; * *$ 表示极显著性差别 $(p$ $<0.01$ )。

The relative concentration of each metabolite is the average of data from five biological replicates obtained through gas chromatography-mass spectrometry. Changes were calculated using the formula $\log _{2}$ (treatment/control). * indicates significant $(p<0.05)$ and $* *$ highly significant difference $(p<0.01)$, respectively.

存在拮抗竞争(Parida \& Das, 2005)。本研究结果显 示，碱胁迫下根系中 $\mathrm{Na}$ 含量增加的同时造成K含量 减少，这表明碱胁迫影响根质膜中 $\mathrm{Na}^{+} / \mathrm{H}^{+}$交换活性， 减少细胞质内 $\mathrm{Na}^{+}$排放的同时阻碍 $\mathrm{K}^{+}$吸收和运输, 最终导致小麦体内离子和 $\mathrm{pH}$ 平衡遭到破坏( $\mathrm{Zhu}$, 2003; Munns \& Tester, 2008)。与以往对盐胁迫的研
究结果刚好相反, 碱胁迫下Ca大量积累, 这可能触 发SOS-Na系统，形成SOS2-SOS3 激酶复合体提高 $S O S 1$ 活性，促进 $\mathrm{Na}^{+}$外排减少毒害作用(Wang et al., 2011; Wu et al., 2013; 郭瑞等, 2016)。碱胁迫下根系 中 $\mathrm{Na}$ 含量的激增不仅直接引起 $\mathrm{Mg} 、 \mathrm{Cu} 、 \mathrm{Fe}$ 和 $\mathrm{Zn}$ 含 量降低, 还严重影响 $\mathrm{Cl}^{-} 、 \mathrm{NO}_{3}{ }^{-}$和 $\mathrm{H}_{2} \mathrm{PO}_{4}{ }^{-}$积累, 这说 www.plant-ecology.com 
明碱胁迫不仅影响矿质元素代谢，还强烈干扰无机 阴离子吸收、积累和分布, 加深对植物的毒害作用 (杨春武等, 2008)。

\section{2 碱胁迫对小麦根系代谢产物的影响}

本研究利用GC-MS代谢组学分析为碱胁迫下 植物的响应提供丰富的生化信息, 全面系统描述代 谢物组成成分的变化情况以及涉及的植物适应碱胁 迫的重要代谢途径。研究结果表明, 碱胁迫处理使 小麦产生了显著的代谢变化, 包括三羧酸循环、糖 酵解/糖异生和氨基酸等代谢过程。本研究比之前文
献报道的碱胁迫下植物的生理生化改变显示出更为 全面的、具体的和复杂的代谢变化，而且这种代谢 途径的变化可能与植物对高 $\mathrm{pH}$ 伤害有直接的关系 (郭伟和于立河, 2012; 胡海燕等, 2013)。

与对照组相比, 碱胁迫下小麦根系代谢响应变 化明显, 糖酵解、细胞膜脂代谢和氨基酸合成受到 明显的抑制，但TCA循环显著增强(图3)。结果表明 小麦根系中合成代谢活性受到高 $\mathrm{pH}$ 影响非常大，而 能量代谢对于小麦适应高 $\mathrm{pH}$ 环境起到至关重要的 作用, 它不仅是碱胁迫下小麦根系的 N-C转变过程,

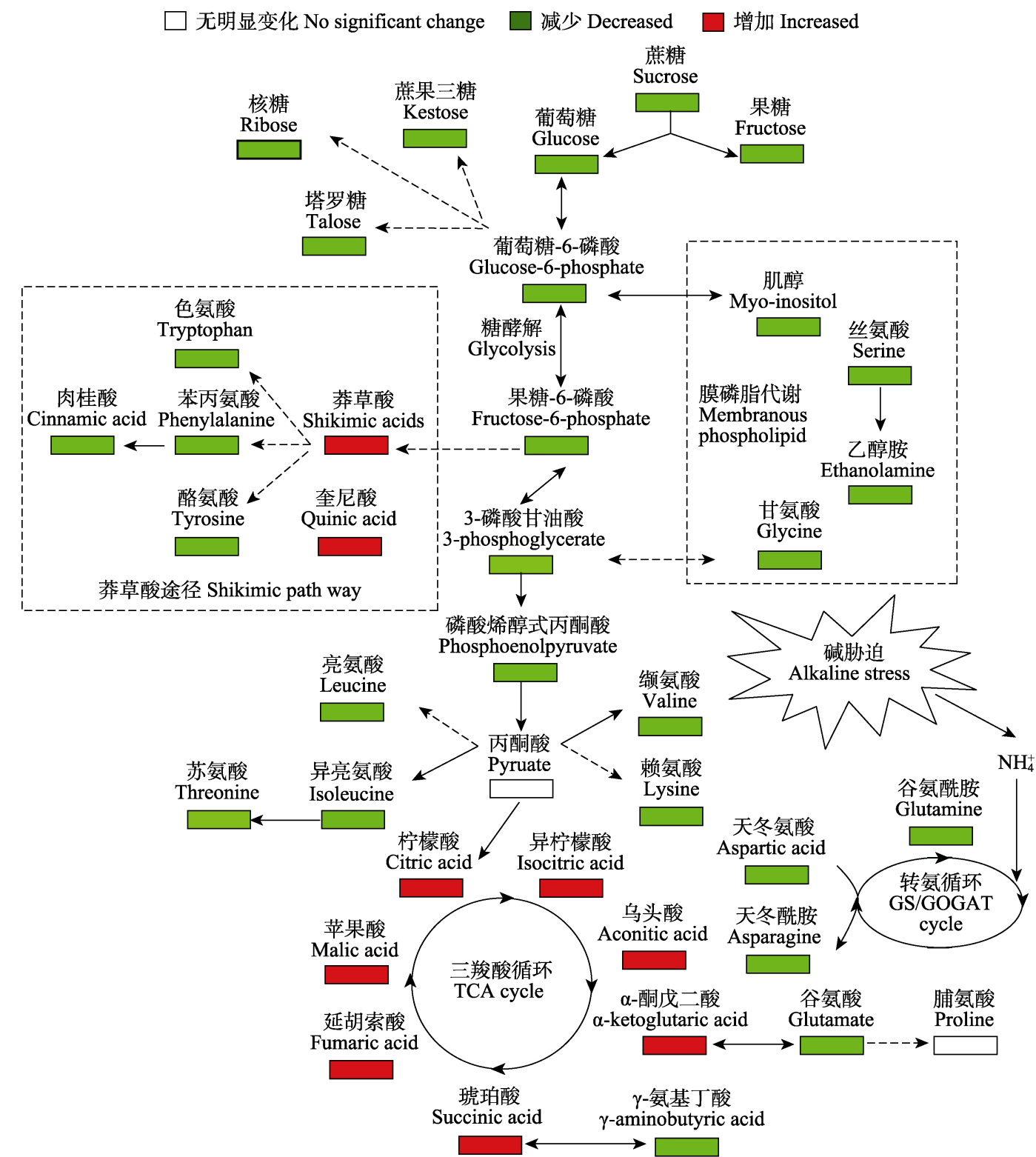

图3 OPLS-DA分析得出的碱胁迫下小麦根系代谢途径网络变化图。白色代表无明显变化, 红色代表显著性增加, 绿色代表 显著性降低 $(p<0.05)$ 。

Fig. 3 Proposed metabolic network changes for wheat roots upon alkaline stress obtained from OPLS-DA analysis. The metabolite with white boxes denotes no significant change while red boxes denotes significant increases and green ones denotes significant decreases $(p<0.05)$. 
还为细胞的各种化合物生物合成提供所需能量, 以 维持渗透压平衡、减少离子毒害作用、调节 $\mathrm{pH}$ 值。 另外, 碱胁迫下根系有机酸大量积累可能是对无机 阴离子亏缺的响应, 当大量 $\mathrm{Na}^{+}$涌入植物体内造成 总正电荷激增, 同时, 阴离子 $\left(\mathrm{Cl}^{-} 、 \mathrm{NO}_{3}{ }^{-} 、 \mathrm{H}_{2} \mathrm{PO}_{4}{ }^{-}\right)$ 的大量减少造成负电荷严重亏缺, 小麦根系中有机 酸可以弥补无机负电荷不足、保持细胞内 $\mathrm{pH}$ 稳定 (表3; 图3)。所以, 有机酸不仅是能量代谢的重要中 间产物, 还是维持细胞内 $\mathrm{pH}$ 稳定和离子平衡的重要 响应机制。糖类是植物体生命活动最主要的能源物 质, 主要来自光合作用, 糖异生途径和多糖降解(王 镜岩等, 2002)。本研究发现, 碱胁迫迫使小麦根系 糖类及多元醇含量显著下降, 结合Yang等(2008)对 碱胁迫下小麦光合速率显著下降的研究结果, 发现 碱胁迫对糖类的合成和储存有明显的负效应, 在高 $\mathrm{pH}$ 环境下植物细胞中 $\mathrm{Na}^{+}$大量积累造成离子毒害, 不仅破坏光合器官, 而且对糖酵解途径也产生了严 重的抑制作用, 这意味着高 $\mathrm{pH}$ 使得植物体本身清除 活性氧(ROS)的能力明显下降。结果还表明, 糖类合 成和积累不是一个简单的被动胁迫响应, 而是对高 $\mathrm{pH}$ 极为敏感的主动代谢调控过程。

高等植物受到水分缺失或盐胁迫时通常会积 累大量的脯氨酸, 来保护细胞膜和蛋白质以及清除 活性氧, 在植物抗逆生理中起到非常重要的作用 (彭志红等, 2002, Wu et al., 2013; 姜淑欣等, 2014)。 本研究发现碱胁迫没有引起脯氨酸的明显积累, 脯 氨酸含量显著低于其他有机渗透调节物质如柠檬酸 和苹果酸。结果表明碱胁迫可能抑制谷氨酸介导的 脯氨酸生物合成途径(图3), 因此碱胁迫下的脯氨酸 更可能作为信号分子或者充当蛋白酶的激活剂, 而 并非作为渗透调节物质, 这有待于进一步深入研究 (Kerepesi \& Galiba, 2000; Parvaneh et al., 2012)。转 氨基产物(谷氨酰胺、天冬酰胺、天冬氨酸含量的降 低)可能通过氨基作用生成 $\alpha$-酮戊二酸进入TCA循 环, 这不仅可以缓解碱胁迫引起的过量 $\mathrm{NH}_{4}{ }^{+}$对小麦 根系造成的“毒性”, 可以促进 TCA循环, 为生成有 机酸类化合物提供能量来源, 这可能是小麦适应高 $\mathrm{pH}$ 的一种特殊相应对策。 $\gamma$-氨基丁酸(GABA)是重要 的 $\mathrm{N}$ 代谢中间产物, 在调节渗透势和 $\mathrm{pH}$ 值以及 $\mathrm{TCA}$ 循环回补作用方面起着重要作用(Parida \& Das, 2005)。一般情况下, 植物在干旱或热胁迫环境中积 累大量GABA, 但本研究发现碱胁迫导致其含量显
著降低, 结合丙氨酸的大量减少, 以及琥珀酸显著 增加, 研究结果暗示植物可能通过GABA旁路代谢 途径(GABA shunt)回补琥珀酸, 进而促进TCA循 环。支链氨基酸(缅氨酸、异亮氨酸和亮氨酸)的明 显减少进一步说明碱胁迫对氨基酸代谢的抑制作 用。在小麦根系中碱胁迫引起氨基酸含量减少的主 要原因可能是由于高 $\mathrm{pH}$ 对氮代谢的影响。植物对氮 素的利用主要是通过根系质膜上的特定蛋白AMT (ammonium transporter)和NRT (nitrate transporter)吸 收铵态氮 $\left(\mathrm{NH}_{4}{ }^{+}\right)$和硝态氮 $\left(\mathrm{NO}_{3}{ }^{-}\right)$, 由跨质膜的质子 电化学梯度提供能量, 并通过感知自身的氮素水平 调控相关代谢过程(邓若否等, 2007; 骆媛媛和柳参 奎, 2009)。碱胁迫破坏离子平衡造成外部质子缺乏, 从而削弱 AMT和 NRT活性, 使得 $\mathrm{NH}_{4}{ }^{+}$和 $\mathrm{NO}_{3}{ }^{-}$吸收 受阻。本研究发现高 $\mathrm{pH}$ 胁迫下小麦根系中 $\mathrm{NO}_{3}{ }^{-}$含量 几乎为 0 , 氨基酸含量下降明显, 这些结果证明了以 上结论(表3; 图3)。

\section{4 结论}

碱胁迫下根系中 $\mathrm{Na}$ 大量积累加之高 $\mathrm{pH}$ 环境, 造成 $\mathrm{K}$ 含量骤降, 抑制矿质元素吸收, 同时造成阴 离子吸收受阻导致离子严重失衡, 使得植物生长受 阻甚至死亡。碱胁迫对根系代谢产物及相关代谢途 径产生很大的影响, 结果表明碱胁迫造成小麦根系 中糖类和氨基酸类代谢物含量显著降低, 表明碱胁 迫下糖酵解和氨基酸代谢途径受到明显抑制; 同时 碱胁迫限制 $\mathrm{C}-\mathrm{N}$ 转变过程影响小麦对 $\mathrm{N}$ 素的利用, 造成营养贵乏抑制植物生长发育。碱胁迫严重影响 细胞膜脂代谢, 破坏细胞膜造成根系功能障碍, 进 一步加剧碱胁迫对植物体的伤害。但碱胁迫促进了 $\mathrm{TCA}$ 循环途径, 为生成有机酸类化合物提供能量来 源。说明小麦为缓解碱胁迫伤害合成大量有机酸, 用来调节体内 $\mathrm{pH}$ 值和维持离子平衡，以维持正常的 生理代谢过程, 这可能是小麦适应高pH的一种特殊 相应对策。

\section{基金项目 国家自然科学基金(31200243 和 31570328)。}

\section{参考文献}

Deng RL, Xu HR, Cao YF, Xiao K (2007). The molecular basis of ammonium transporters in plants. Plant Nutrition and Fertilizer Science, 13, 512-519. (in Chinese with English 
abstract) [邓若䂞, 徐海荣, 曹云飞, 肖凯 (2007). 植物 吸收铵态氮的分子生物学基础. 植物营养与肥料学报, 13, 512-519.]

FAO (2005). Global network on integrated soil management for sustainable use of salt affected soil. http://www.fao.org/ag/ agl/agll/spush. Cited: 2016-03-28

Guo R, Li F, Zhou J, Li HR, Xia X, Liu Q (2016). Eco-physiological responses of linseed (Linum usitatissimum) to salt and alkali stresses. Chinese Journal of Plant Ecology, 40, 69-79. (in Chinese with English abstract) [郭瑞, 李峰, 周际, 李昊儒, 夏旭, 刘琪 (2016). 亚麻响应盐、碱胁迫 的生理特征. 植物生态学报, 40, 69-79.]

Guo W, Yu LH (2012). Effects of salinity-alkalinity stress on root activity and phenylalanine ammonia-lyase activity of wheat seedlings. Crops, (1), 31-34. (in Chinese with English abstract) [郭伟, 于立河 (2012). 盐碱胁迫对小麦幼 苗根系活力和苯丙氨酸解氨酶活性的影响. 作物杂志, (1), 31-34.]

Hu HY, He J, Zhao JJ, Ru ZG (2013). Dynamic change of cell defense enzyme activity in wheat seedling under different alkaline $\mathrm{pH}$ conditions. Journal of Henan Institute of Science and Technology (Natural Science Edition), 41(5), 1-5. (in Chinese with English abstract) [ 胡海燕, 贺杰, 赵 俊杰, 茹振刚 (2013). 碱性pH条件下小麦幼苗保护酶 活性的变化动态. 河南科技学院学报(自然科学版), 41(5), 1-5.]

Jianaer AH, Yang CW, Shi DC, Wang DL (2007). Physiological response of an alkali resistant halophyte Kochia sieversiana to salt and alkali stresses. Acta Botanica Boreali-Occidentalia Sinica, 27, 79-84. (in Chinese with English abstract) [贾娜尔 - 阿汗, 杨春武, 石德成, 王德利 (2007). 盐生植物碱地肤对盐碱胁迫的生理响应特点. 西北植物学报, 27, 79-84. ]

Jiang SX, Liu DX, Pang HX, Lü JY (2014). Effects of PEG stress and recovery on activities of key enzymes involved in proline metabolism in wheat cultivars with difference in drought tolerance. Acta Botanica Boreali-Occidentalia Sinica, 34, 1581-1587. (in Chinese with English abstract) [姜淑欣, 刘党校, 庞红喜, 吕金印 (2014). PEG胁迫及 复水对不同抗旱性小麦幼苗脯氨酸代谢关键酶活性的 影响. 西北植物学报, 34, 1581-1587.]

Kerepesi I, Galiba G (2000). Osmotic and salt stress induced alteration in soluble carbohydrate content in wheat seedlings. Crop Science, 40, 482-487.

Kingsbury RW, Epstein E, Peary RW (1984). Physiological responses to salinity in selected lines of wheat. Plant Physiology, 74, 417-423.

Li B, Wang ZC, Sun ZG, Chen Y, Yang F (2005). Resources and sustainable resource exploitation of salinized land in China. Agricultural Research in the Arid Areas, 23(2), 152-158. (in Chinese with English abstract) [李涁, 王志 春, 孙志高, 陈渊, 杨福 (2005). 中国盐碱地资源与可
持续利用研究. 干旱地区农业研究, 23(2), 152-158.]

Lisec J, Schauer N, Kopka J, Willmitzer L, Fernie AR (2006). Gas chromatography mass spectrometry-based metabolite profiling in plants. Nature Protocol, 1, 387-396.

Luo YY, Liu SK (2009). Research progress of ammonium transporter in plants. Genomics and Applied Biology, 28, 373-379. (in Chinese with English abstract) [骆媛媛, 柳 参奎 (2009). 植物中铵转运蛋白的研究进展. 基因组学 与应用生物学, 28, 373-379.]

Munns R, Tester M (2008). Mechanisms of salinity tolerance. Annual Review of Plant Biology, 59, 651-681.

Parida AK, Das AB (2005). Salt tolerance and salinity effects on plants: A review. Ecotoxicology and Environmental Safety, 60, 324-349.

Parvaneh R, Shahrokh T, Hosseini SM (2012). Studying of salinity stress effect on germination, proline, sugar, protein, lipid and chlorophyll content in purslane (Portulace oloracea L.) leaves. Stress Physiology \& Biochemistry, 8, 182-193.

Peng ZH, Peng KQ, Hu JJ, Xiao LT (2002). Research progress on accumulation of proline under osmotic stress in plants. Chinese Agriculture Science Bulletin, 18(4), 80-83. (in Chinese with English abstract) [彭志红，彭克勤，胡家金， 萧浪涛 (2002). 渗透胁迫下植物脯氨酸积累的研究进 展. 中国农学通报, 18(4), 80-83.]

Sa RL, Liu JH, Liu L, Bai JH, Wang ZH (2014). Cation- responsive mechanisms of oats to alkali stress. Acta Agronomica Sinica, 40, 362-368. (in Chinese with English abstract) [萨如拉, 刘景辉, 刘伟, 白健慧, 王占海 (2014). 燕麦对碱胁迫的阳离子响应机制. 作物学报, 40, 362-368.]

Shi DC, Wang DL (2005). Effects of various salt-alkaline mixed stresses on Aneurolepidium chinense (Trin.) Kitag. Plant and Soil, 271, 15-26.

Shi LX, Ma S, Fang Y, Xu J (2015). Crucial variations in growth and ion homeostasis of glycine gracilis seedlings under two types of salt stresses. Journal of Soil Science and Plant Nutrition, 15, 1-17.

Wang H, Wu ZH, Han JY, Zheng W, Yang CW (2012). Comparison of ion balance and nitrogen metabolism in old and young leaves of alkali-stressed rice plants. PLOS ONE, 7, e37817. doi: 10.1371/journal.pone.0037817.

Wang JY, Zhu SG, Xu CF (2002). Biochemistry. 3rd edn. Higher Education Press, Beijing. (in Chinese) [王镜岩, 朱 圣庚, 徐长法 (2002). 生物化学. 第三版. 高等教育出 版社, 北京.]

Wang XD, Wang C, Ma ZH, Hou RF, Gao Q, Chen Q (2011). Effect of short-term salt stress on the absorption of $\mathrm{K}^{+}$and accumulation of $\mathrm{Na}^{+}, \mathrm{K}^{+}$in seedlings of different wheat varieties. Acta Ecologica Sinica, 31, 2822-2830. (in Chinese with English abstract) [王晓冬, 王成, 马智宏, 侯瑞 锋, 高权, 陈泉 (2011). 短期 $\mathrm{NaCl}$ 胁迫对不同小麦品种 
幼苗 $\mathrm{K}^{+}$吸收和 $\mathrm{Na}^{+} 、 \mathrm{~K}^{+}$积累的影响. 生态学报, 31 , 2822-2830.]

Wang XP, Geng SJ, Ri YJ, Cao DH, Liu J, Shi DC, Yang CW (2011). Physiological responses and adaptive strategies of tomato plants to salt and alkali stresses. Scientia Horticulturae, 130, 248-255.

Wu DZ, Shen QF, Cai SG, Chen ZH, Dai F, Zhang GP (2013). Ionomic responses and correlations between elements and metabolites under salt stress in wild and cultivated barley. Plant \& Cell Physiology, 54, 1976-1988.

Yang CW, Li CY, Zhang ML, Liu J, Ju M, Shi DC (2008). pH and ion balance in wheat-wheatgrass under salt-or alkali stress. Chinese Journal of Applied Ecology, 19, 10001005. (in Chinese with English abstract) [杨春武, 李长有, 张美丽, 刘杰, 鞠沝, 石德成 (2008). 盐、碱胁迫下小冰 麦体内的 $\mathrm{pH}$ 及离子平衡. 应用生态学报, 19, 10001005.]

Yang C, Shi D, Wang D (2008). Comparative effects of salt stress and alkali stress on growth, osmotic adjustment and ionic balance of an alkali resistant halophyte Suaeda glauca (Bge.). Plant Growth Regulation, 56, 179-190.

Yang T, Xie ZX, Yu Q, Liu XJ (2014). Effects of partial root salt stress on seedling growth and photosynthetic characteristics of winter wheat. Chinese Journal of Eco- Agrculture, 22, 1074-1078. (in Chinese with English abstract) [杨婷, 谢志霞, 喻琼, 刘小京 (2014). 局部根系盐胁迫 对冬小麦生长和光合特征的影响. 中国生态农业学报, 22, 1074-1078.]

Yu RP (1999). Saline soil resources in China and their exploitation. Chinese Journal of Soil Science, 30(4), 158-159. (in Chinese with English abstract) [俞仁培 (1999). 我国盐 渍土资源及其开发利用. 土壤通报, 30(4), 158-159.]

Zhu JK (2003). Regulation of ion homeostasis under salt stress. Current Opinion in Plant Biology, 6, 441-445.

责任编委: 王 雷 责任编辑: 李 敏

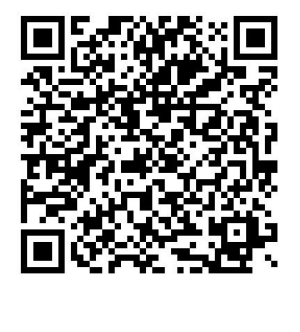

